PROCEEDINGS OF THE

AMERICAN MATHEMATICAL SOCIETY

Volume 126, Number 7, July 1998, Pages 1915-1921

S 0002-9939(98)04391-3

\title{
DERIVED LENGTHS AND CHARACTER DEGREES
}

\author{
MARK L. LEWIS \\ (Communicated by Ronald M. Solomon)
}

\begin{abstract}
Let $G$ be a finite solvable group. Assume that the degree graph of $G$ has exactly two connected components that do not contain 1. Suppose that one of these connected components contains the subset $\left\{a_{1}, \ldots, a_{n}\right\}$, where $a_{i}$ and $a_{j}$ are coprime when $i \neq j$. Then the derived length of $G$ is less than or equal to $|\operatorname{cd}(G)|-n+1$.
\end{abstract}

\section{INTRODUCTION}

In the theory of solvable groups, a long-standing conjecture of G. Seitz says that if $G$ is a finite solvable group, then $\operatorname{dl}(G) \leq|\operatorname{cd}(G)|$, where $\operatorname{dl}(G)$ is the derived length of $G$ and $\operatorname{cd}(G)$ is the set of distinct irreducible character degrees of $G$. For $M$-groups this is just Taketa's theorem (Corollary 5.13 of $[4]$ ). When $|\operatorname{cd}(G)|=2$, 3 , or 4, this has been verified in Corollary 12.6 of [4], Theorem 12.15 of [4], and [2], respectively. Berger showed that the conjecture is true for groups having odd order (see Corollary 16.7 of [9]). In this paper, we offer further evidence that this conjecture is true.

The situation of interest involves the character degree graph of a group. We defined the degree graph $\Gamma(G)$ of $G$ in $[6]$ to be the graph having $\operatorname{cd}(G)$ as vertices and with two character degrees being adjacent if they have a common divisor larger than 1 . Note that 1 is an isolated vertex in this graph. We proved in [6] that when $G$ is a solvable group this graph has at most two more connected components. In this paper, our interest is in those solvable groups where both of these connected components are not empty. This case has been studied in several places. In particular, this situation is the subject of Section 19 of [9]. (Note that their degree graph is not the same graph as ours. However, it is not difficult to prove that the number of connected components in their graph is equal to the number of connected components in our graph that do not contain 1.) This situation is also the subject of the papers [8] and [10]. (In the terminology of those papers, the group is said to be a character $\pi$-separable group, where $\pi$ is the set of primes dividing the degrees in one of the connected components of the graph.)

Theorem A. Let $G$ be a solvable group. Assume that $\Gamma(G)$ has exactly two connected components not containing 1 . Then $\operatorname{dl}(G) \leq|\operatorname{cd}(G)|$.

It is a widely held belief that the derived length of a solvable group is actually strictly less than $|\operatorname{cd}(G)|$ when $|\operatorname{cd}(G)|$ is large enough (i.e., greater than 4). In

Received by the editors December 16, 1996.

1991 Mathematics Subject Classification. Primary 20C15.

(C)1998 American Mathematical Society 
our case, we will show that with an additional piece of information we can break through this barrier.

Theorem B. Let $G$ be a solvable group. Assume that $\Gamma(G)$ has exactly two connected components not containing 1 . Furthermore, suppose that one of the connected components of $\Gamma(G)$ has a subset $\left\{a_{1}, a_{2}, \ldots, a_{n}\right\}$ where $a_{i}$ and $a_{j}$ are coprime when $i \neq j$. Then $\operatorname{dl}(G) \leq|\operatorname{cd}(G)|-n+1$.

Observe that Theorem A is a corollary of Theorem B. In particular, since there are exactly two connected components not containing 1 , we can choose a character degree $a_{1}$ in one of them, and then apply Theorem B with $n=1$.

In proving Theorem $\mathrm{B}$, we need to consider an action where all the nonlinear irreducible characters are invariant. That this situation arises in our case is not a surprise, since it seems to occur whenever one wants to consider solvable groups $G$ such that $\Gamma(G)$ has exactly two connected components that do not contain 1 (see Section 19 of [9]). In this situation, we prove the following theorem. Note that the content of this theorem is the bound on the derived length, since Isaacs proved in Theorem A of [5] that the group $N$ in this theorem is solvable.

Theorem C. Let $H$ act nontrivially via automorphisms on $N$ fixing every nonlinear irreducible character, where $(|H|,|N|)=1$. Then $N$ is a solvable group satisfying $\mathrm{dl}(N) \leq|\operatorname{cd}(N)|$.

We note that the class of groups that are covered by the hypotheses of these theorems is very restrictive. In particular, if $G$ is a group satisfying the hypotheses of Theorem A, then the Fitting height of $G$ is between 2 and 4 and the derived length of $G / F$ is less than or equal to 4 , where $F$ is the Fitting subgroup of $G$ (see Theorem 19.6 of [9]). Also, in Theorem 3.3 of [5] (or Theorem 19.3 of [9]), Isaacs found a classification for those groups that satisfy the hypotheses of Theorem C. Despite this, we believe that our result has merit, since both classes contain groups of arbitrarily large derived length. For those groups satisfying Theorem C, a class of examples can be found in Theorem 4.9 of [5]. In Section 4, we will use this class of examples to prove that there exist groups of arbitrarily large derived length that satisfy the hypotheses of Theorem A.

We would like to conclude this introduction by thanking Professor Gagola for pointing out that this result might be possible and for his many helpful conversations while working out the result. Also, we would like to thank the referee for pointing out a nice simplification in the proof of Lemma 1.

\section{ACTIONS FIXING EVERY NONLINEAR IRREDUCIBLE CHARACTER}

In this section, we consider an action where every nonlinear irreducible character is fixed. When this action is coprime, we are in the situation of Theorem C. To prove Theorem $\mathrm{B}$, we use a generalization of Theorem $\mathrm{C}$ where the action is not necessarily coprime. To that end, we present the following technical lemma. Note that Theorem $\mathrm{C}$ is just the case in this lemma when $N$ is coprime to $H$. It is easy to see that the extra hypotheses found in this lemma are satisfied in the case of Theorem C. If Theorem $\mathrm{C}$ were the only result needed from this lemma, the proof would be quite a bit shorter, i.e., it would consist only of the first two steps of our proof.

Lemma 1. Let $\pi$ be a set of primes. Suppose that a $\pi$-group $H$ acts via automorphisms on a $\pi$-separable group $N$ such that $H$ acts nontrivially on $N / \mathbf{O}^{\pi^{\prime}}(N)$. 
Furthermore, assume that every nonlinear irreducible character of $N$ has $\pi^{\prime}$-degree and is invariant under the action of $H$. Then $N$ is solvable, and $\operatorname{dl}(N) \leq|\operatorname{cd}(N)|$.

Proof. By a theorem of Gallagher in [1], since every irreducible character of $N$ has $\pi^{\prime}$-degree it can be shown that $N$ has a normal abelian Hall $\pi$-subgroup $Q$. This implies that $\mathbf{O}^{\pi^{\prime}}(N)=Q$. Now, $H$ acts coprimely and nontrivially on $N / Q$, fixing every nonlinear irreducible character. By Theorem A of [5], $N / Q$ is solvable, and hence $N$ is solvable. We now work to show that $\operatorname{dl}(N) \leq|\operatorname{cd}(N)|$.

We suppose that this result is not true, and we take $N$ to be a counterexample with $|N|$ minimal. That is, $N$ is a solvable group with the property that $\operatorname{dl}(N)>$ $|\operatorname{cd}(N)|$. In particular, $N$ is not an $M$-group.

Step 1. $[N, H] Q / Q$ is a Frobenius group with kernel $[N, H]^{\prime} Q / Q=N^{\prime} Q / Q$.

Proof of Step 1. As we observed before, $H$ acts coprimely and nontrivially on $N / Q$, fixing every nonlinear irreducible character. We are now in the situation of Theorem 19.3 of [9]. From that theorem, we know that $N^{\prime} Q=[N, H]^{\prime} Q$ and that one of the following holds: (1) $[N, H] Q / Q$ is abelian, (2) $[N, H] Q / Q$ is a class $2 p$-group for some prime $p$, or (3) $[N, H] Q / Q$ is a Frobenius group with kernel $N^{\prime} Q / Q$.

Assume we are in Case (1), so that $[N, H] Q / Q$ is abelian. This implies that $[N, H]^{\prime} \subseteq Q$. Since $N^{\prime} Q=[N, H]^{\prime} Q$, it follows that $N^{\prime} \subseteq Q$. We know that $Q$ is an abelian Hall subgroup and $N / Q$ is abelian, so it follows that every Sylow subgroup of $N$ is abelian. From Theorem 6.23 of [4], $N$ is now an $M$-group, contradicting the choice of $N$. Thus, we are not in Case (1).

Assume now that we are in Case (2), so that $[N, H] Q / Q$ is a class $2 p$-group for some prime $p$. Write $C / N^{\prime} Q=\mathbf{C}_{N / N^{\prime} Q}(H)$. Since $N^{\prime} \subseteq C$, we have that $C$ is normal in $N$. Observe that $N=[N, H] C$. This implies that $N / C$ is isomorphic to the group $[N, H] Q /([N, H] Q \cap C)$. Since $C$ contains $N^{\prime} Q$, we see that $[N, H] Q /([N, H] Q \cap C)$ is a quotient of $[N, H] Q / Q$, and so it follows that $N / C$ is a $p$-group. If $C=N$, then $H$ fixes every irreducible character of $N / Q$. By Lemma 12.2 of [9], $H$ centralizes $N / Q$, in contradiction to the hypothesis that $H$ acts nontrivially on $N / Q$. Therefore, we conclude that $C<N$. Observe that $H$ fixes only the identity coset of $N / C$. Since $N / C$ is abelian, $H$ acts on the conjugacy classes of $N / C$, fixing only the identity conjugacy class. By a theorem of Brauer, Theorem 6.32 of [4], $H$ fixes the same number of characters in $\operatorname{Irr}(N / C)$ as the number of conjugacy classes of $N / C$. Thus, $H$ fixes only the principal character of $N / C$. Consider characters $\nu \in \operatorname{Irr}(N)$ with $\nu(1)>1$ and $\lambda \in \operatorname{Irr}(N / C)$. Since $\nu$ and $\nu \lambda$ are nonlinear irreducible characters of $N$, they are both invariant under the action of $H$. Thus, we have that $\nu \lambda=(\nu \lambda)^{h}=\nu \lambda^{h}$ for every element $h \in H$. Rewriting this equation, we obtain the following: $\nu=\nu \lambda^{-1} \lambda^{h}$. This implies that $\nu$ must take the value 0 on those elements outside the kernel of $[\lambda, h]$. Because every character in $\operatorname{Irr}(N / C)$ has this form, this implies that the vanishing-off subgroup of $\nu$ is contained in $C$. Since $N / C$ is a $p$-group, it follows that $p$ divides $\nu(1)$. This is true for every nonlinear character of $N$. Thus, by a theorem of Thompson (Corollary 12.2 of [4]), $N$ has a normal $p$-complement $M$.

Observe that $Q \subseteq M$ and $M \cap[N, H] Q=Q$. Hence, $M / Q$ is isomorphic to the group $[N, H] M /[N, H] Q$. Since $N^{\prime} \subseteq[N, H] Q$, we have that $M / Q$ is abelian. As in Case (1), this implies that every Sylow subgroup of $M$ is abelian. Because $N / M$ is a $p$-group, we apply Theorem 6.22 of [4] to see that $N$ is a relative $M$-group with respect to $M$. From Theorem 6.23 of [4], we conclude that $N$ is an $M$-group, 
in contradiction to the choice of $N$. Therefore, we are left with Case (3), which is the desired result.

Step 2. If $M \neq 1$ is an abelian subgroup of $N$ which is normal in $N H$, then $\operatorname{cd}(N / M)=\operatorname{cd}(N)$. Furthermore, $N H$ has a unique minimal normal subgroup contained in $N$.

Proof of Step 2. By Step 1, we know that $[N, H] Q / Q$ is not an abelian group, and so we cannot have that $[N, H] \subseteq M Q$. Therefore, the action of $H$ on $N / M Q$ is still nontrivial. Hence, we may apply the induction hypothesis to $N / M$ to see that $\operatorname{dl}(N / M) \leq|\operatorname{cd}(N / M)|$. Since $M$ is abelian, it follows that $\operatorname{dl}(N) \leq|\operatorname{cd}(N / M)|+1$. If $\operatorname{dl}(N)=\operatorname{dl}(N / M)$, then $\operatorname{dl}(N) \leq|\operatorname{cd}(N / M)| \leq|\operatorname{cd}(N)|$, contradicting the choice of $N$. Because $\operatorname{dl}(N) \leq \operatorname{dl}(N / M)+1$, we conclude that $\operatorname{dl}(N)=\operatorname{dl}(N / M)+1$. If $\operatorname{cd}(N)>\operatorname{cd}(N / M)$, then $|\operatorname{cd}(N)| \geq|\operatorname{cd}(N / M)|+1 \geq \operatorname{dl}(N / M)+1=\operatorname{dl}(N)$. Again this contradicts the choice of $N$, so we have that $\operatorname{cd}(N)=\operatorname{cd}(N / M)$.

Suppose now that $M_{1}$ and $M_{2}$ are distinct minimal normal subgroups of $N H$ contained in $N$. Take $m=|\operatorname{cd}(N / M)|$, so that from the previous paragraph we see that $N^{(m)} \subseteq M_{i}$ for $i=1,2$, where $N^{(m)}$ is the $m$ th derived subgroup of $N$. This implies that $N^{m} \subseteq M_{1} \cap M_{2}=1$. Therefore, we conclude that $\operatorname{dl}(N) \leq m \leq$ $|\operatorname{cd}(N)|$, which is a contradiction to the choice of $N$, and so we have completed this step.

We now show that $Q>1$. In the application of this lemma to the situation where $N$ is a $\pi^{\prime}$-group, Step 2 completes the proof. In general, $N$ need not be a $\pi^{\prime}$-group.

Step 3. $Q>1$.

Proof of Step 3. Assume that $Q=1$. By Step 1, $N^{\prime}$ is a Frobenius kernel, and hence it is nilpotent by a theorem of Thompson, Satz V.8.7 of [3]. From Step 2, we know that $N H$ has a unique minimal normal subgroup contained in $N$. It follows that $N^{\prime}$ has a unique subgroup that is minimal normal in $N H$, and this can only happen when $N^{\prime}$ is a $p$-group for some prime $p$.

Let $P$ be a Sylow $p$ subgroup of $N$. Since $N^{\prime}$ is a normal $p$-subgroup, we see that $N^{\prime} \subseteq P$, and so $P$ is normal in $N$. Consider characters $\varphi \in \operatorname{Irr}(P)$ and $\nu \in \operatorname{Irr}(N \mid \varphi)$. Observe that $\nu(1)_{p}=\varphi(1)$. If $\gamma \in \operatorname{Irr}(P)$ with $\gamma(1) \neq \varphi(1)$ and $\mu \in \operatorname{Irr}(N \mid \gamma)$, then $\mu(1)_{p}=\gamma(1) \neq \varphi(1)=\nu(1)_{p}$. It follows that $\mu(1) \neq \nu(1)$ and that $|\operatorname{cd}(N)| \geq|\operatorname{cd}(P)|$. Since $P$ is a $p$-group, we know that $\operatorname{dl}(P) \leq|\operatorname{cd}(P)|$. We have that $P^{\prime} \subseteq N^{\prime}$. If $P^{\prime}=N^{\prime}$, then $\operatorname{dl}(N)=\operatorname{dl}(P) \leq|\operatorname{cd}(P)| \leq|\operatorname{cd}(N)|$, in contradiction to the choice of $N$. This implies that $P^{\prime}<N^{\prime}$. Thus, there is a linear character $\lambda \in \operatorname{Irr}(P)$ so that $\lambda_{N^{\prime}} \neq 1_{N^{\prime}}$. Since $[N, H]$ is a Frobenius group with kernel $N^{\prime}, \lambda_{N^{\prime}}$ lies in an $[N, H]$-orbit of size $\left|[N, H]: N^{\prime}\right|$. It follows that $\lambda$ lies in an orbit of size divisible by $\left|[N, H]: N^{\prime}\right|$. If the character $\nu \in \operatorname{Irr}(P \mid \lambda)$, then $\nu(1) \neq 1$ and $\nu(1)_{p}=\lambda(1)=1$. We conclude that $|\operatorname{cd}(N)| \geq|\operatorname{cd}(P)|+1 \geq \operatorname{dl}(P)+1 \geq \operatorname{dl}(N)$, which contradicts the choice of $N$. Therefore, $Q>1$.

Step 4. $Q$ is the Fitting subgroup of $N$.

Proof of Step 4. Consider a Hall $\pi^{\prime}$-subgroup $B$ of $\mathbf{C}_{N}(Q)$. Since $B$ centralizes $Q$, it follows that $B$ is normal in $\mathbf{C}_{N}(Q)$, and so $B$ is characteristic in $\mathbf{C}_{N}(Q)$. Observe that $\mathbf{C}_{N}(Q)$ is normal in $N$. Also, since $H$ normalizes both $N$ and $Q$, we determine that $H$ normalizes $\mathbf{C}_{N}(Q)$. Therefore, $\mathbf{C}_{N}(Q)$ is normal in $N H$, and so 
we conclude that $B$ is normal in $N$. By Step 3, we know that $Q$ is nontrivial. This implies that $Q$ contains a minimal normal subgroup of $N H$. From Step 2, this is the only minimal normal subgroup of $N H$ in $N$, and hence $B$ does not contain any minimal normal subgroup of $N H$. This can only occur if $B=1$. Therefore, we have that $Q=\mathbf{C}_{N}(Q)$. Since the Hall $\pi$-complement of the Fitting subgroup of $N$ must centralize $Q$, we conclude that $Q$ is the Fitting subgroup of $N$.

Step 5. Final contradiction.

Proof of Step 5. Since $Q$ is abelian, we apply Step 2, to see that $\operatorname{cd}(N)=\operatorname{cd}(N / Q)$. From Step 3, we know that $Q>1$, and applying the induction hypothesis to $N / Q$, we see that $\operatorname{dl}(N / Q) \leq|\operatorname{cd}(N / Q)|$. We use the fact from Step 4 that $Q$ is the Fitting subgroup of $N$ along with a theorem of Broline and Garrison, Theorem 12.19 of [4], to see that there is at least one character degree in the set $\operatorname{cd}(N)$ that is not in the set $\operatorname{cd}(N / Q)$. Therefore, we have that $\operatorname{dl}(N) \leq \operatorname{dl}(N / Q)+1 \leq|\operatorname{cd}(N / Q)|+1 \leq$ $|\operatorname{cd}(N)|$. This contradicts the choice of $N$, and proves the lemma.

\section{Proof of Theorem B}

We are now prepared to prove Theorem B. The argument found here was motivated by the simplified version found in Lemma 2.4 of [7].

Proof of Theorem B. Let $K$ be a subgroup of $G$ that is maximal with the property that $K$ is normal in $G$ and $G / K$ is nonabelian. Since $\Gamma(G)$ has exactly two connected components that do not contain 1, it is easy to see that $G / K$ is a Frobenius group with cyclic complement and elementary abelian $p$-group kernel $N / K$ for some prime $p$. (A proof of this result can be found as Lemma 2.3 of [7].) Write $f=|G: N|$, so that $\operatorname{cd}(G / K)=\{1, f\}$. Let $X$ and $Y$ be the connected components of $\Gamma(G)$ that do not contain 1. Without loss of generality, we may assume that $f \in Y$. From Theorem 12.4 of [4], we know that $p$ divides every element in $X$. Therefore, if $n>1$, then the set $\left\{a_{1}, a_{2}, \ldots, a_{n}\right\}$ must be a subset of $Y$.

Consider a character $\chi \in \operatorname{Irr}(G)$ such that $\chi(1) \in X$. We know that $\chi(1)$ is relatively prime to $f=|G: N|$, and so $\chi_{N} \in \operatorname{Irr}(N)$. This implies that $X \subseteq \operatorname{cd}(N)$. Now, suppose we have a character $\theta \in \operatorname{Irr}(N)$ such that $\theta(1) \in X$. When we pick a character $\chi \in \operatorname{Irr}(G \mid \theta)$, it is clear that $\chi(1) \in X$, and so $\chi_{N}=\theta$. Thus, every irreducible character of $N$ having degree in $X$ extends to $G$. On the other hand, assume that the character $\theta \in \operatorname{Irr}(N)$ has $\theta(1) \neq 1$ and $\theta(1) \notin X$. Thus, if we look at a character $\chi \in \operatorname{Irr}(G \mid \theta)$, then we observe that $\chi(1) \in Y$. This implies that $p$ does not divide $\theta(1)$, and so, by Theorem 12.4 of [4], we know that $\theta(1) f \in \operatorname{Irr}(G)$. Therefore, we see that $\operatorname{cd}(N)=1 \cup X \cup Y^{*}$, where $Y^{*}=\{a \in \operatorname{cd}(N) \mid a \neq 1, a f \in$ $\operatorname{cd}(G)\}$. It is not hard to determine that $X$ and $Y^{*}$ are connected components for $\Gamma(N)$. We note that the map $a \mapsto a f$ is a one-to-one function from $Y^{*}$ to $Y$. Since $1 \notin Y^{*}$, it follows that $f$ is not in the image of $Y^{*}$ under this map. Also, when $n>1$, at most one of the $a_{i}$ is divisible by $f$, so the remaining $n-1$ of the $a_{i}$ are not in the image of $Y^{*}$ under this map. Regardless of whether $n=1$ or $n>1$, we conclude that $\left|Y^{*}\right| \leq|Y|-n$. Since $\operatorname{cd}(G)=1 \cup X \cup Y$, it follows that $\operatorname{cd}(N) \leq \operatorname{cd}(G)-n$. If $\left|Y^{*}\right| \geq 1$, then there exists an integer $a_{1} \in Y^{*}$, and so the hypotheses of the theorem hold for $N$ with $n=1$. By the induction hypothesis, we see that $\operatorname{dl}(G) \leq \operatorname{dl}(N)+1 \leq(|\operatorname{cd}(N)|-1+1)+1 \leq(|\operatorname{cd}(G)|-n)+1$, and the result holds in this case. Thus, we have that $\left|Y^{*}\right|=0$, and we conclude that $\operatorname{cd}(N)=1 \cup X$. 
Take $\pi$ to be the set of primes dividing integers in $Y$, and write $H$ for a Hall $\pi$ subgroup of $G$. Thus, $H$ is a $\pi$-group acting on $N$. Since every nonlinear irreducible character of $N$ has degree lying in $X$, it is extendible to $G$. Hence, we see that the action of $H$ fixes every nonlinear irreducible character of $N$ and that every irreducible character of $N$ has $\pi^{\prime}$-degree. Since the action of $H /(H \cap N)$ on $N / K$ is a Frobenius action and since $N / K$ is a $\pi^{\prime}$-group, the action of $H$ on $N / \mathbf{O}^{\pi^{\prime}}(N)$ is nontrivial. We are now in the situation of Lemma 1. From that lemma we have that

$$
\operatorname{dl}(G) \leq \operatorname{dl}(N)+1 \leq|\operatorname{cd}(N)|+1 \leq|\operatorname{cd}(G)|-n+1,
$$

proving the theorem.

\section{Arbitrarily LARGE DERIVED LENGTHS}

We now use the examples found in Theorem 4.9 of [5] to show that there exist groups $G$ of arbitrarily large derived length that satisfy the hypotheses of Theorem A.

Lemma 2. Given a prime number $p$ and an integer $n \geq 1$, choose a prime number $m>\max (p, n)$. Then there is a group $G$ such that one connected component of $\Gamma(G)$ has the isolated vertex $m$, another connected component of $\Gamma(G)$ consists of powers of $p$, and $\mathrm{dl}(G)>\log _{2}(n)$.

Proof. Take $N$ and $H$ to be groups that satisfy Theorem 4.9 of [5], so that $H$ is a cyclic group of order $m$ acting coprimely on the $p$-group $N$ fixing every nonlinear irreducible character of $N$. That theorem also tells us that $H$ fixes none of the nontrivial linear characters of $N$ and that $\operatorname{dl}(N)>\log _{2}(n)$. Now take $G$ to be the semi-direct product of $H$ acting on $N$, so that $\operatorname{dl}(G) \geq \operatorname{dl}(N)>\log _{2}(n)$. Because $H$ is cyclic and every nonlinear character of $N$ is $H$-invariant, it follows that every irreducible character of $G$ whose restriction to $N$ contains a nonlinear irreducible constituent must have $p$-power degree. On the other hand, we may use Ito's theorem (Theorem 6.15 of [4]) to see that every irreducible character of $G / N^{\prime}$ has degree dividing $m$. The fact that $m$ occurs in $\operatorname{cd}(G)$ follows since $H$ fixes no nontrivial linear character of $N$. Therefore, we conclude that $\operatorname{cd}(G)=$ $\{1\} \cup\{m\} \cup \operatorname{cd}(N)$, as desired.

\section{REFERENCES}

[1] P. X. Gallagher, Group characters and normal Hall subgroups, Nagoya Math. J. 21 (1962), 223-230. MR 26:240

[2] S. C. Garrison, On groups with a small number of character degrees, Ph.D. Thesis, Univ. of Wisconsin, Madison, 1973.

[3] B. Huppert, Endlichen gruppen I, Springer-Verlag, Berlin, 1967. MR 37:302

[4] I. M. Isaacs, Character theory of finite groups, Academic Press, New York, 1976. MR 57:417

[5] I. M. Isaacs, Coprime group actions fixing all nonlinear irreducible characters, Can. J. Math. 41 (1989), 68-82. MR 90j:20038

[6] M. L. Lewis, Solvable groups having almost relatively prime distinct irreducible character degrees, J. Algebra 174 (1995), 197-216. MR 96d:20009

[7] M. L. Lewis, Derived lengths of solvable groups satisfying the one-prime hypothesis, Submitted to Comm. in Algebra.

[8] O. Manz and R. Staszewski, Some applications of a fundamental theorem by Gluck and Wolf in the character theory of finite groups, Math. Z. 192 (1986), 383-389. MR 87i:20018 
[9] O. Manz and T. R. Wolf, Representations of solvable groups, Cambridge University Press, Cambridge, 1993. MR 95c:20013

[10] R. Staszewski, On $\pi$-blocks of finite groups, Comm. in Algebra 13 (1985), 2369-2405. MR 86j:20011

Department of Mathematics and Computer Science, Kent State University, Kent, Оніо 44242

E-mail address: lewis@mcs.kent.edu 